\title{
Komplementäre, alternative und integrative Therapien in den medizinischen Leitlinien - die Leitlinie "Diagnostik und Therapie der Colitis ulcerosa" als Beispiel gelungener Integration
}

\author{
Holger Cramer ${ }^{a}$ Romy Lauche ${ }^{a}$ Petra Klose $^{a}$ Gustav Dobos ${ }^{a}$ Jost Langhorst ${ }^{a, b}$ \\ ${ }^{a}$ Klinik für Naturheilkunde und Integrative Medizin, \\ ${ }^{\mathrm{b}}$ Zentrum für Integrative Gastroenterologie, Kliniken Essen-Mitte, Medizinische Fakultät, Universität Duisburg-Essen, Deutschland
}

Die aktuelle Leitlinie «Diagnostik und Therapie der Colitis ulcerosa» wurde im September 2011 unter Federführung der Deutschen Gesellschaft für Verdauungs- und Stoffwechselkrankheiten (DGVS) und Mitarbeit der Deutschen Gesellschaft für Pathologie (DGP), der Deutschen Gesellschaft für Allgemein- und Viszeralchirurgie (DGVC), der Gesellschaft für pädiatrische Gastroenterologie und Ernährung (GPGE) sowie der Deutschen Gesellschaft für Ernährungsmedizin (DGEM) vorgelegt. Die nächste Aktualisierung ist für September 2016 avisiert; die gegebenen Empfehlungen werden also noch eine geraume Weile den Goldstandard in der Therapie der Colitis ulcerosa darstellen [1].

Für eine Reihe komplementärmedizinischer Verfahren enthielt die Leitlinie evidenzbasierte positive Empfehlungen, die einen hinreichenden Konsens in der Leitlinienkonferenz fanden (Tab. 1). So wurden abgeschwächt positive Empfehlungen für das Phytotherapeutikum Plantago ovata gegeben, das sich in einer klinischen Studie Mesalazin in der remissionserhaltenden Behandlung als ebenbürtig erwies (Empfehlung 10.16); ebenso für Curcumin, das in der remissionserhaltenden Behandlung komplementär zu 5-ASA empfohlen wird (Empfehlung 10.15). Ein Problem in der Anwendung von Curcumin in Deutschland ergibt sich allerdings daraus, dass in der japanischen Studie [2], auf der die Empfehlung beruht, Curcumin in einer Dosis von $2 \times 1 \mathrm{~g}$ /Tag eingesetzt wurde, während die in Deutschland verfügbaren Präparate deutlich geringere Dosierungen enthalten. Daneben gibt die Leitlinie eine abgeschwächt positive Empfehlung für Akupunktur in Kombination mit Moxibustion als komplementäre Therapie im leichten bis moderaten Schub (Empfehlung 10.18) sowie für eine multimodale komplementäre Mind-Body-Therapie zur Verbesserung der Lebensqualität (Empfehlung 10.17). Letztere Empfehlung ist bisher nahezu einzigartig in Deutschland; erstmals wird hier eine multimodale Mind-Body-Therapie empfohlen, wie sie in Deutschland in integrativmedizinischen Kliniken angeboten wird [3]. Die Empfehlung basiert unter anderem auf einer deutschen randomisierten Studie $[4,5]$, die zeigen konnte, dass ein multimodales Lebensstilmodifikationsprogramm mit Elementen der Mind-Body-Medizin die Lebensqualität von Patienten in Remission oder mit geringer Krankheitsaktivität in klinisch relevantem Umfang steigern kann. Weitere komplementärmedizinische Verfahren, inklusive weiterer Phytotherapeutika und einzelner Verfahren der Mind-Body-Medizin wie Yoga oder Qigong, konnten aufgrund der unzureichenden Datenlage nicht empfohlen werden (Empfehlung 10.20). Ein potenzieller Kandidat für zukünftige Aktualisierungen der Leitlinie scheint die Behandlung mit Eiern des Schweinepeitschenbandwurms (Trichuris suis ovata) zu sein, die in einer randomisierten Studie im leichten bis moderaten Schub die Krankheitsaktivität signifikant stärker senken konnte als Placebo, derzeit aber - vor dem Hintergrund der fehlenden Zulassung in Deutschland - nicht generell empfohlen wird (Empfehlung 10.19). Aktuell wird je eine europäische und eine nordamerikanische Multicenterstudie zur Effektivität von Trichuris suis ovata bei chronisch entzündlichen Darmerkrankungen durchgeführt - allerdings nur bei Patienten mit Morbus Crohn.

Die Empfehlung einer Anzahl komplementärmedizinischer Verfahren in der Therapie der Colitis ulcerosa bietet denjenigen Behandlern Sicherheit, die diese Verfahren aufgrund klinischer Erfahrungen und positiver Studienergebnisse bereits anwenden oder eine Anwendung erwägen, und kann auch konventionell arbeitende Kollegen ermutigen, sich intensiver mit diesen Verfahren zu beschäftigen. Die Leitlinie geht jedoch noch einen deutlichen Schritt weiter und kann hier als

\section{KARGER}

Fax +497614520714

Information@Karger.com

www.karger.com (c) 2014 S. Karger GmbH, Freiburg

$1661-4119 / 14 / 0211-0004 \$ 39.50 / 0$

Accessible online at:

www.karger.com/fok
Prof. Dr. med. Jost Langhorst

Klinik für Naturheilkunde und Integrative Medizin

Kliniken Essen-Mitte, Knappschafts-Krankenhaus

Am Deimelsberg 34a, 45276 Essen, Deutschland

j.langhorst@kliniken-essen-mitte.de 
Tab. 1. Komplementärmedizinische Verfahren in der Leitlinie «Colitis ulcerosa»

\begin{tabular}{|c|c|c|c|c|}
\hline Therapie & Ziel & Evidenzgrad $^{\mathrm{a}}$ & Konsensusstärke & Empfehlung \\
\hline Curcumin & $\begin{array}{l}\text { Remissionserhaltung komplementär } \\
\text { zu 5-ASA }\end{array}$ & B & 75-95\% Zustimmung & $\begin{array}{l}\text { abgeschwächt positiv } \\
\text { («probably do it») }\end{array}$ \\
\hline Plantago ovata & Remissionserhaltung & $\mathrm{B}$ & 75-95\% Zustimmung & abgeschwächt positiv \\
\hline Mind-Body-Therapie & Verbesserung der Lebensqualität & $\mathrm{B}$ & 75-95\% Zustimmung & abgeschwächt positiv \\
\hline Akupunktur (mit Moxibustion) & Senkung der Krankheitsaktivität & $\mathrm{B}$ & 75-95\% Zustimmung & abgeschwächt positiv \\
\hline Trichuris suis ovata & Senkung der Krankheitsaktivität & $\mathrm{B}$ & $>95 \%$ Zustimmung & unklar \\
\hline
\end{tabular}

${ }^{a}$ Evidenzgrad gemäß «Levels of evidence and grades of recommendation» des Oxford Centre for Evidence-Based Medicine [8].

Botschafter einer gelungenen Integration der Komplementärmedizin in die Gastroenterologie aufgefasst werden:Trotz ausdrücklich fehlender Evidenz für diese Forderung gibt die Leitlinie eine starke Empfehlung dafür ab, als behandelnder Arzt die Patienten über die Anwendung komplementärer Heilmethoden zu befragen und über ihre Gründe für die Anwendung zu sprechen (Empfehlung 10.14). Dass diese Empfehlung notwendig ist, zeigt sich schon daran, dass in Deutschland mehr als die Hälfte der Patienten mit chronisch entzündlichen Darmerkrankungen komplementäre Therapieverfahren selbständig anwenden [6,7]. Dabei werden insbesondere die klassischen Verfahren Homöopathie, Phytotherapie und Akupunktur verwendet, häufig auch mehrere Verfahren parallel. Ein Teil der Patienten informiert den behandelnden Arzt nicht über die Nutzung solcher Verfahren, sodass Wechselwirkungen nicht eingeschätzt werden können - es sei denn, der Arzt fragt explizit danach. Doch nicht nur reden soll man mit den Patienten über komplementäre Therapien; eine (eingeschränkte) Empfehlung wird auch gegeben, sich «aufgrund des hohen Anteils an Patienten, die komplementärmedizinische Therapien anwenden, (...) über diese Verfahren (zu) informieren» (Empfehlung 10.21). Schließlich kann die Angemessenheit eines durch die Patienten gewählten Verfahrens sonst kaum eingeschätzt werden. Im Kommentar zu dieser Empfehlung regt die Leitlinie sogar dazu an, dass der konventionelle Mediziner auf Nachfrage seiner Patienten erwägen solle, solche Verfahren selbst in den Behandlungsplan zu integrieren. Für eine medizinische Leitlinie geradezu revolutionär mutet der daraus folgende Kommentar an, der das Kapitel zu Komplementär- und Alternativmedizin abschließt: «Idealerweise ist ein integratives Konzept anzustreben, bei dem konventionelle und komplementäre Therapieverfahren $\mathrm{zu}$ einer <est practice〉 verschmelzen (WHO-Definition von integrativer Medizin)» (Empfehlung 10.21). Die integrative Medizin ist also in den medizinischen Leitlinien angekommen.

Eine besondere Stärke der Leitlinie liegt weiterhin darin, dass sie die Unterscheidung zwischen alternativen und konventionellen Verfahren nicht theoriegeleitet trifft, sondern sich gänzlich auf die verfügbare Evidenz stützt. So wird zwar der alleinige Einsatz alternativer, d.h. nicht evidenzbasierter Therapien abgelehnt, allerdings werden etwa Phytotherapeutika wie Plantago ovata, deren Äquipotenz zu chemischen Arzneimitteln in klinischen Studien belegt wurde, ausdrücklich nicht als alternative Heilmittel angesehen (Empfehlung 10.12). Diese Einschätzung kann als richtungweisend für die gesamte Diskussion um komplementäre und alternative Verfahren in den medizinischen Leitlinien angesehen werden: Die meisten Leser der FORSCHENDEN KOMPLEMENTÄRMEDIZIN werden der Einschätzung zustimmen, dass Verfahren ohne hinreichenden Wirkungsnachweis unter keinen Umständen alternativ zu wirkungsvollen Verfahren eingesetzt werden dürfen. Sobald allerdings der Wirkungsnachweis für ein unkonventionelles Verfahren erbracht ist, kann und muss es in die Patientenversorgung eingebracht werden - komplementär oder auch alternativ zu etablierten Therapien. Ein positives Beispiel für dieses Vorgehen findet sich etwa bei den Empfehlungen zu Probiotika. Probiotika haben seit Langem ihren festen Platz in der Komplementärmedizin. Aufgrund einer hinreichenden Evidenzbasierung erscheinen die positiven Statements zu Probiotika nun in den Leitlinienkapiteln «Remissionserhaltung» (Empfehlung 5.15) und «Chirurgie» (Empfehlung 7.26) statt im Kapitel «Komplementär- und Alternativmedizin».WeitereForschungzukomplementär-undalternativmedizinischen Verfahren wird in der Leitlinie aufgrund des großen öffentlichen Interesses ausdrücklich eingefordert. Dabei wird den Besonderheiten vieler komplementärmedizinischer Verfahren Rechnung getragen, deren komplexe Struktur, intensive Arzt-Patienten-Beziehung und Multimodalität durch klassische randomisierte Studien häufig nicht hinreichend abgebildet werden können. Stattdessen wird in der Leitlinie ausdrücklich vorgeschlagen, bei der Evaluation solcher komplexen Verfahren die Ergebnisse von Kohortenstudien in der «Evidence-based medicine»-Hierarchisierung als gleichwertig zu denen randomisierter Studien anzusehen (Empfehlung 10.13). Namentlich wird die weitere Evaluation von Verfahren wie TCM, Ayurveda, manuellen Therapien, Aromatherapie, Nahrungsergänzungsmitteln, Mind-Body-Medizin und auch polarisierender Verfahren wie Reiki und Homöopathie angeregt (Empfehlung 10.12). Ein nobler Grundsatz für die Entwicklung medizinischer Leitlinien, der Schule machen sollte ist: Sich ausschließlich auf die wissenschaftliche Evidenz zu stützen statt auf a priori gebildete Überzeugungen, und sogar solchen Verfahren eine faire Chance zu geben, die zwar im Kanon der etablierten Medizin bisher wenig Berücksichtigung finden, dafür aber von den Betroffenen umso mehr nachgefragt werden. 


\section{Literatur}

1 Dignass A, Preiß JC, Aust DE, Autschbach F, Ballauff A, Barretton G, Bokemeyer B, Fichtner-Feig S, Hagel S, Herrlinger KR, Jantschek G, Kroesen A, Kruis W, Kucharzik T, Langhorst J, Reinshagen M, Rogler G, Schleiermacher D, Schmidt C, Schreiber S, Schulze H, Stange E, Zeitz M, Hoffmann JC Stallmach A: Aktualisierte Leitlinie zur Diagnostik und Therapie der Colitis ulcerosa 2011 - Ergebnisse einer evidenzbasierten Konsensuskonferenz. Z Gastroenterol 2011;49:1276-1341.

2 Hanai H, Iida T, Takeuchi K, Watanabe F, Maruyama Y, Andoh A, Tsujikawa T, Fujiyama Y, Mitsuyama K, Sata M, Yamada M, Iwaoka Y, Kanke K, Hiraishi H, Hirayama K, Arai H, Yoshii S, Uchijima M, Nagata T, Koide Y: Curcumin maintenance therapy for ulcerative colitis: randomized, multicenter, double-blind, placebo-controlled trial. Clin Gastroenterol Hepatol 2006;4:1502-1506.
Paul A, Lauche R, Cramer H, Altner N, Dobos G: An integrative day-care clinic for chronically ill patients: concept and case presentation. Eur J Integr Med 2012;4:E455-E459.

4 Elsenbruch S, Langhorst J, Popkirowa K, Müller T, Lüdtke R, Franken U, Paul A, Spahn G, Michalsen A, Janssen OE, Schedlowski M, Dobos GJ: Effects of mind-body therapy on quality of life and neuroendocrine and cellular immune functions in patients with ulcerative colitis. Psychother Psychosom 2005;74:277-287.

5 Langhorst J, Müller T, Lüdtke R, Franken U, Paul A, Michalsen A, Schedlowski M, Dobos GJ, Elsenbruch S: Effects of a comprehensive lifestyle modification program on quality-of-life in patients with ulcerative colitis: a twelve-month follow-up. Scand J Gastroenterol 2007;42:734-745.
6 Langhorst J, Anthonisen JB, Steder-Neukamm U, Lüdtke R, Spahn G, Michalsen A, Dobos GJ: Amount of systemic steroid medication is a strong predictor for the use of complementary and alternative medicine in patients with inflammatory bowel disease: results from a German national survey. Inflamm Bowel Dis 2005;11:287-295.

7 Langhorst J, Anthonisen JB, Steder-Neukamm U, Lüdtke R, Spahn G, Michalsen A, Dobos GJ: Patterns of complementary and alternative medicine (CAM) use in patients with inflammatory bowel disease: perceived stress is a potential indicator for CAM use. Complement Ther Med 2007;15:30-37.

8 Oxford Centre for Evidence-based Medicine: Levels of evidence (March 2009). www.cebm.net/?o=1025 (accessed 13.01.2014). 